Title : will be set by the publisher

Editors: will be set by the publisher

EAS Publications Series, Vol. ?, 2013

\title{
COMBISPEC: A NOVEL APPROACH IN THE MODELLING OF MASSIVE BINARIES
}

\author{
M. Palate ${ }^{1}$
}

\begin{abstract}
Spectral modelling and especially for massive stars is not a straightforward problem and over the past decades many advances have been made in this field. We now have very efficient model atmosphere codes such as CMFGEN and TLUSTY for example. However, these codes are based on the assumption that the star is single and spherical which is no longer valid for the components of a binary system. On the other hand, observational studies indicate that binarity has an impact on the spectra and binary interactions impact the analyses of the spectra. This is why we have developed a very first model called CoMBiSpeC (code of massive binary spectral computation). We present here some examples of the impact of the binarity on the shape of the stars and the resulting effects on their spectra.
\end{abstract}

\section{Introduction}

(Massive) binary systems are crucial to improve our knowledge of the fundamental properties of (massive) stars. One of common way to derive these properties is synthetic spectral modelling. However, the current stellar atmosphere models are designed for single spherical stars and therefore neglect interaction effects that occur in close binaries. In this context I have developed the CoMBiSpeC (code of massive binary spectral computation) model that account for the interactions between the stars in the spectral computation. CoMBiSpeC computes the stellar surface of circular synchronous rotating system and then the gravity and temperature distribution as well as the spectra at several orbital phases. In the case of eccentric system the TIDES (Tidal interactions with dissipation of energy through shear) code (Moreno et al. 1999, 2005, 2011) is used for the surface and velocity field computation. Our model is presented in Sect. 2 and three examples of application are described in Sect. 3.

\footnotetext{
${ }^{1}$ Institut d'Astrophysique et de Géophysique, Université de Liège, Bât. B5c, Allée du 6 Août 17, 4000 Liège, Belgium
} 


\section{Model}

For clarity, I divided my code CoMBiSpeC into two parts, one part involving the surface, gravity and temperature calculation, and a second the spectral computation. A full description of the model can be found in Palate \& Rauw (2012) and Palate et al. (2013a).

\subsection{Geometrical modelling}

In the case of circular systems in synchronous rotation, the stellar surface is an equipotential of the Roche potential. In massive stars, the radiation pressure is very important and acts on the shape of stars, I thus have modified the classical Roche potential by adding the internal radiation pressure (effect on each star's own surface) and the external radiation pressure (effect of the companion) effects. A more detailed description of the radiation pressure effect can be found in Palate et al. (2013a). Our approach is based on the works of Drechsel et al. (1995) and Phillips \& Podsiadlowski (2002) for the treatment of the external radiation pressure and the Howarth (1997) approach for the inner radiation pressure treatment. The effect of the external radiation pressure can be seen as a force that decreases the attraction of the companion. Its treatment therefore consists of scaling the mass of the companion in the Roche potential. The scale parameter $\delta=\frac{F_{\text {rad }}}{F_{\text {grav }}}$ is computed iteratively for each surface point in a similar way to the reflection effect treatment of Wilson (1990). The modified "Roche" potential can be written (for the primary)

$$
\Omega=\frac{1}{r}+\frac{q(1-\delta(r, \varphi, \theta))}{\sqrt{r^{2}-2 r \cos \varphi \sin \theta+1}}+\frac{q+1}{2} \cdot r^{2} \sin ^{2} \theta-q r \cos \varphi \sin \theta,
$$

where $r=\sqrt{x^{2}+y^{2}+z^{2}}, q=\frac{m_{2}}{m_{1}}, x=r \cos \varphi \sin \theta, y=r \sin \varphi \sin \theta$, and $z=r \cos \theta$. Here, $\theta$ and $\varphi$ are, respectively, the colatitude and longitude angle in the spherical coordinates centred on the star under consideration.

According to Howarth (1997), the inner radiation pressure can be treated as a simple scaling of the Roche potential and thus, $\Omega_{e f f}=(1-\Gamma) \Omega$, with $\Gamma=\frac{\sigma_{T h}}{m_{h} c} \sigma T_{\text {pole }}^{4} \frac{1}{\| \vec{p} \text { pole }} \|$ and where $\frac{\sigma_{T h}}{m_{H}} \approx 0.036 \mathrm{~m}^{2} \mathrm{~kg}^{-1}, \sigma_{T h}$ is the Thomson scattering cross section and $\sigma$ the Stefan-Boltzmann constant. The stellar surfaces are represented with a discretised grid of $240 \times 60$ points (in $\varphi$ and $\theta$ respectively). The local acceleration of gravity is given by the gradient of the Roche potential. The temperature is computed accounting for gravity darkening and following the von Zeipel ((1924)) theorem

$$
T_{\text {local }}=T_{\text {pole }}\left(\frac{\left\|\underline{\nabla} \Omega_{\text {local }}\right\|}{\left\|\underline{\nabla} \Omega_{\text {pole }}\right\|}\right)^{0.25},
$$

We also account for the reflection effects described by Wilson (1990) that leads to a local increase in temperature of the front part of the stars due to the companion.

In the cases of eccentric binaries, we used the TIDES code (Moreno et al. 1999, 2005, 2011) for the surface and velocity field computation. The TIDES code calculation involves the solution of the equations of motion of the surface elements in the presence of gravitational, Coriolis, centrifugal, viscous and gas pressure forces. We then used 
CoMBiSpeC to complete the geometrical computation (i.e. the gravity and temperature computation).

\subsection{Spectral modelling}

The second part of the algorithm computes the spectrum of the binary by summing the incremental contributions of each surface point. Non-LTE OB star spectral grids (TLUSTY OSTAR2002 and BSTAR2006 grid, Lanz \& Hubeny 2003, 2007) are used to compute the integrated spectrum of the star at each orbital phase. The spectral grid is computed for solar metallicity. Each spectrum is defined by two parameters: gravity and temperature. As we know these parameters for each point at the stellar surfaces, we can compute the local contribution to the spectrum. The computation consists of a linear interpolation between the flux of the four nearest spectra in the grid. The appropriate Doppler shift is then applied to the spectrum accounting for the orbital and rotational velocity of the surface element. We multiply the spectrum by the area of the element projected along the line of sight towards the observer and by a limb-darkening coefficient based on the tabulation of Claret \& Bloemen (2011). Finally, we sum the contribution to the total spectrum. It has to be stressed that we have assumed that there is no heat transfer between the different surface elements as far as the formation of the spectrum is concerned. In the case of circular orbit, phase zero corresponds to the "eclipse" of the primary by the secondary. Over the first half of the orbital cycle (phase $=[0,0.5[$ ), the primary star has a negative radial velocity. For the eccentric orbit, phase zero correspond to the periastron passage.

\section{Examples of application}

We present here three examples in which the CoMBiSpeC and TIDES (for the third example) codes have highlighted new explanations. A more extensive description of these examples can be found in Palate \& Rauw (2012) and Palate et al. (2013a, 2013b). The binary systems used are based on the parameters of two real systems: HD 100213 and HD 165052 studied by Linder et al. (2007). The third example is based on the best parameters derived with the CoMBiSpeC and TIDES codes for the Spica system (see Harrington et al. 2009 and Palate et al. 2013b). The parameters of these systems can be found in Table 1 and 2.

\subsection{Temperature distribution effect}

The model of HD 100213 leads to a contact binary if radiation pressure is not included, but when it is included, the system becomes slightly detached. A non-uniform surface temperature distribution in HD 100213 was reported by Linder et al. (2007). This temperature distribution is observed through radial velocity measurements of the He II and He I lines (see Table 3) that were not formed in the same region of the stellar surfaces. The He II lines are preferentially formed over the hotter region of the stars whilst the He I lines are preferentially formed over the cooler region of the stars. The radial velocity amplitude of the He II lines is smaller which indicates that the low radial velocity regions 
Table 1. Parameters of the circular binary systems. Inclination in brackets stands for non-eclipsing system.

\begin{tabular}{lcc}
\hline \hline Parameters & HD 165052 & HD 100213 \\
\hline Spectral type & O6.5V + O7V & O7.5V + O9.5V \\
Period (day) & 2.95515 & 1.3872 \\
Mass ratio & 0.87 & 0.68 \\
Semi-major axis $\left(R_{\odot}\right)$ & 31.25 & 17.34 \\
Inclination $\left({ }^{\circ}\right)$ & $(23)$ & 77.8 \\
Mass of primary $\left(M_{\odot}\right)$ & 25.15 & 21.7 \\
Mass of secondary $\left(M_{\odot}\right)$ & 21.79 & 14.7 \\
Primary polar temperature $(\mathrm{K})$ & 35500 & 35100 \\
Secondary polar temperature $(\mathrm{K})$ & 34400 & 31500 \\
\hline
\end{tabular}

Table 2. Spica parameters used for computation with the TIDES + CoMBiSpeC model. (a) Argument of periastron of the secondary. (b) The $\beta_{0}$ parameter is the ratio of the rotation and orbital angular velocities at periastron and can be expressed as: $\beta_{0}=0.02 \frac{P v_{\text {rot }}}{R} \frac{(1-e)^{3 / 2}}{(1+e)^{1 / 2}}$, where $v_{\text {rot }}$ is the rigid body rotation velocity (in $\mathrm{km} \mathrm{s}^{-1}$ ), $R$ is the equilibrium radius (in $R_{\odot}$ ), and $e$ is the eccentricity. (c) $1 \mathrm{R}_{\odot}^{2}$ day $^{-1}=5.67 \times 10^{16} \mathrm{~cm}^{2} \mathrm{~s}^{-1}$.

\begin{tabular}{|c|c|c|}
\hline Parameters & Primary & Secondary \\
\hline \multicolumn{3}{|l|}{ Common parameters } \\
\hline Period (day) & \multicolumn{2}{|c|}{4.01452} \\
\hline Eccentricity & \multicolumn{2}{|c|}{0.067} \\
\hline$\omega^{(\mathrm{a})}\left({ }^{\circ}\right)$ & \multicolumn{2}{|c|}{255} \\
\hline Inclination $\left({ }^{\circ}\right)$ & \multicolumn{2}{|c|}{60} \\
\hline \multicolumn{3}{|l|}{ CoMBiSpeC parameters } \\
\hline Mass $\left(M_{\odot}\right)$ & 10.25 & 6.97 \\
\hline Polar temperature $(\mathrm{K})$ & 24000 & 19500 \\
\hline Polar radius $\left(R_{\odot}\right)$ & 6.84 & 3.64 \\
\hline $\mathrm{v}_{\text {rot }}\left(\mathrm{km} \mathrm{s}^{-1}\right)$ & 199 & 87 \\
\hline$\beta_{0}^{(\mathrm{b})}$ & 2.07 & 1.67 \\
\hline Microturbulent velocity $\left(\mathrm{km} \mathrm{s}^{-1}\right)$ & 15 & 15 \\
\hline \multicolumn{3}{|l|}{ TIDES code parameters } \\
\hline Viscosity, $v\left(R_{\odot}^{2} \text { day }^{-1}\right)^{(\mathrm{c})}$ & 0.028 & 0.028 \\
\hline Layer depth $(\Delta R / R)$ & 0.07 & 0.07 \\
\hline Polytropic index & 1.5 & 1.5 \\
\hline
\end{tabular}

have to correspond to the hemisphere facing the companion. The radial velocity amplitude of the He I lines is higher which indicates that the high radial velocity regions have to correspond to the hemisphere opposite to the companion. Without radiation pressure the front hemisphere is too cold to reproduce the temperature distribution, however, when radiation pressure is added, the stars are less deformed. This implies a weak tempera- 
ture increase of the facing hemispheres caused by the decreasing gravity darkening and a higher effectiveness of the reflection process. This weak increase is, therefore, sufficient to explain the observations (within the error bar).

Table 3. Semi-amplitudes of the radial velocity curves (in $\mathrm{km} \mathrm{s}^{-1}$ ) of the He lines in the HD 100213 model spectra.The first two columns are the results obtained with the MIDAS software developed by ESO and the last two are the semi-amplitudes observed by Linder et al. (2007). The error on the observed semi-amplitudes is about $\epsilon \sim \pm 5 \mathrm{~km} \mathrm{~s}^{-1}$ excepted for the Не I $\lambda 5876$ where it is $\epsilon \sim \pm 10$ $\mathrm{km} \mathrm{s}^{-1}$.

\begin{tabular}{lcccc}
\hline \hline Lines & $\mathrm{K}_{1}$ & $\mathrm{~K}_{2}$ & $\mathrm{~K}_{1, \mathrm{obs}}$ & $\mathrm{K}_{2, \mathrm{obs}}$ \\
\hline $\mathrm{He}_{\text {I }} \lambda 4026$ & 245 & 362 & 247 & 364 \\
$\mathrm{He}_{\text {I }} \lambda 4471$ & 246 & 361 & 245 & 372 \\
$\mathrm{He}_{\text {II }} \lambda 4686$ & 241 & 344 & 251 & 355 \\
$\mathrm{He}_{\text {I }} \lambda 4921$ & 257 & 363 & 246 & 373 \\
$\mathrm{He}_{\text {II }} \lambda 5412$ & 239 & 339 & 240 & 351 \\
$\mathrm{He}_{\text {I }} \lambda 5876$ & 245 & 358 & 270 & 389 \\
\hline
\end{tabular}

\subsection{Struve-Sahade effect}

The Struve-Sahade effect (hereafter S-S effect) was originally defined as the apparent strengthening of the secondary spectrum when the star is approaching the observer and its weakening as it moves away. A more general definition is the apparent variation of the line strengths of either of the binary components as a function of the orbital phase (Linder et al. 2007). This effect was reported by Linder et al. (2007) in HD 165052 (e.g. in the $\mathrm{He}_{\mathrm{r}} \lambda 4026$ line). Our simulations show that the S-S effect seems to be due to the combination of the spectra in which the lines have a non-Gaussian/non-Lorentzian and even asymmetric profile. For example, fig. 1 illustrates the $\mathrm{He}_{\mathrm{I}} \lambda 4026$ line at two opposite phases. Our individual spectra reveal this line to be asymmetric with a steeper red wing for both stars. At phase 0.25 , the blue wing of the secondary reinforces the core of the primary that appears to have a higher equivalent width (EW) than its real value. At the phase 0.75 , the situation is inverted and the core of the secondary seems to be reinforced. When the combined binary spectra is fitted with two symmetric Gaussian profiles, the red component will be systematically assigned a lower flux, because the blue component will be apparently broader than the red one. Although the resulting fit can be of excellent quality, it will provide systematically larger EWs for the blue component and smaller EWs for the red. This situation therefore leads to an artificial S-S behaviour. This result hence leads to an alternative explanation of the S-S effect: in some binary systems and at least for some lines, this effect could simply be an artefact caused by the fitting of blended asymmetric lines with symmetric profiles. Therefore, the S-S effect does not stem from genuine physical processes, but rather reflects a bias in the measurement of the line profiles. 

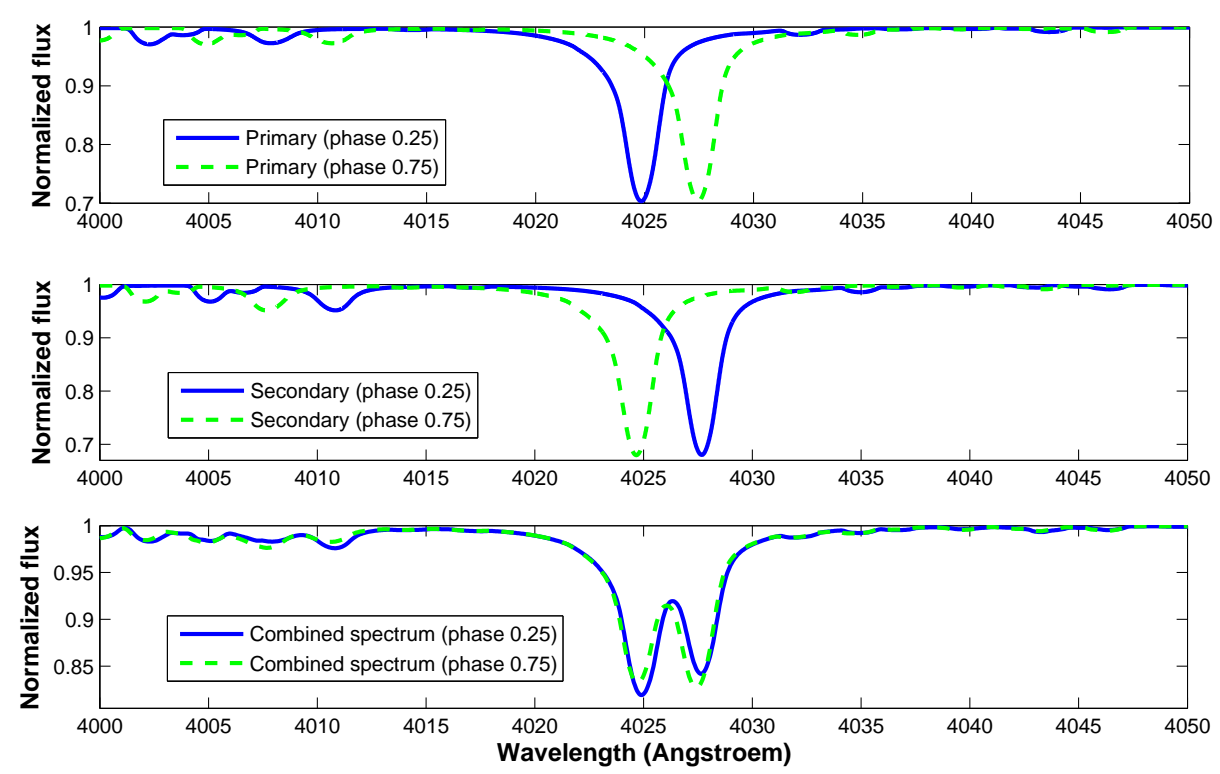

Fig. 1. He I $\lambda 4026$ line at phases 0.25 and 0.75 in the HD 165052 model. Upper panel: Primary spectra. Middle panel: secondary spectra. Lower panel: combined spectra.

\subsection{Line profile variation}

Spica is a double-lined, short-period ( $\sim 4$ days) spectroscopic binary in an eccentric orbit. The primary component is classified as B1.5 IV-V and the secondary component is classified as B3 V. The primary was believed to be a $\beta$ Cephei-type star (Shobbrook et al. 1969, 1972) presenting spectroscopic line-profile variations that are commonly described in terms of travelling "waves" that migrate from the blue to the red wing of the weak photospheric absorption lines, but also include variations in the slope of the line wings.

Harrington et al. (2009) performed an ab initio calculation with the TIDES code of the line profiles at several orbital phases in order to study the variability that is caused by the response of the star to tidal perturbations. They were able to reproduce the general trends in the line-profile variability (migrating "bumps"). Harrington et al. describe these bumps as "tidal flows", a concept that differs from the non-radial pulsation representation in that the travelling waves on the stellar surface are a consequence entirely of the response of this surface to the forcing and restoring agents, the interior structure of the star playing no role. The primary star rotates super-synchronously, which significantly perturbs its surface, leading to strong phase-dependent line profile variations. This super-synchronously rotation explains the presence of the tidal flows.

The study of Harrington et al. (2009) was limited to the analysis of line-profile variability alone, and no comparison of the effects on different lines (particularly those used for temperature and gravity diagnostics) was possible. These effect were modelled with 


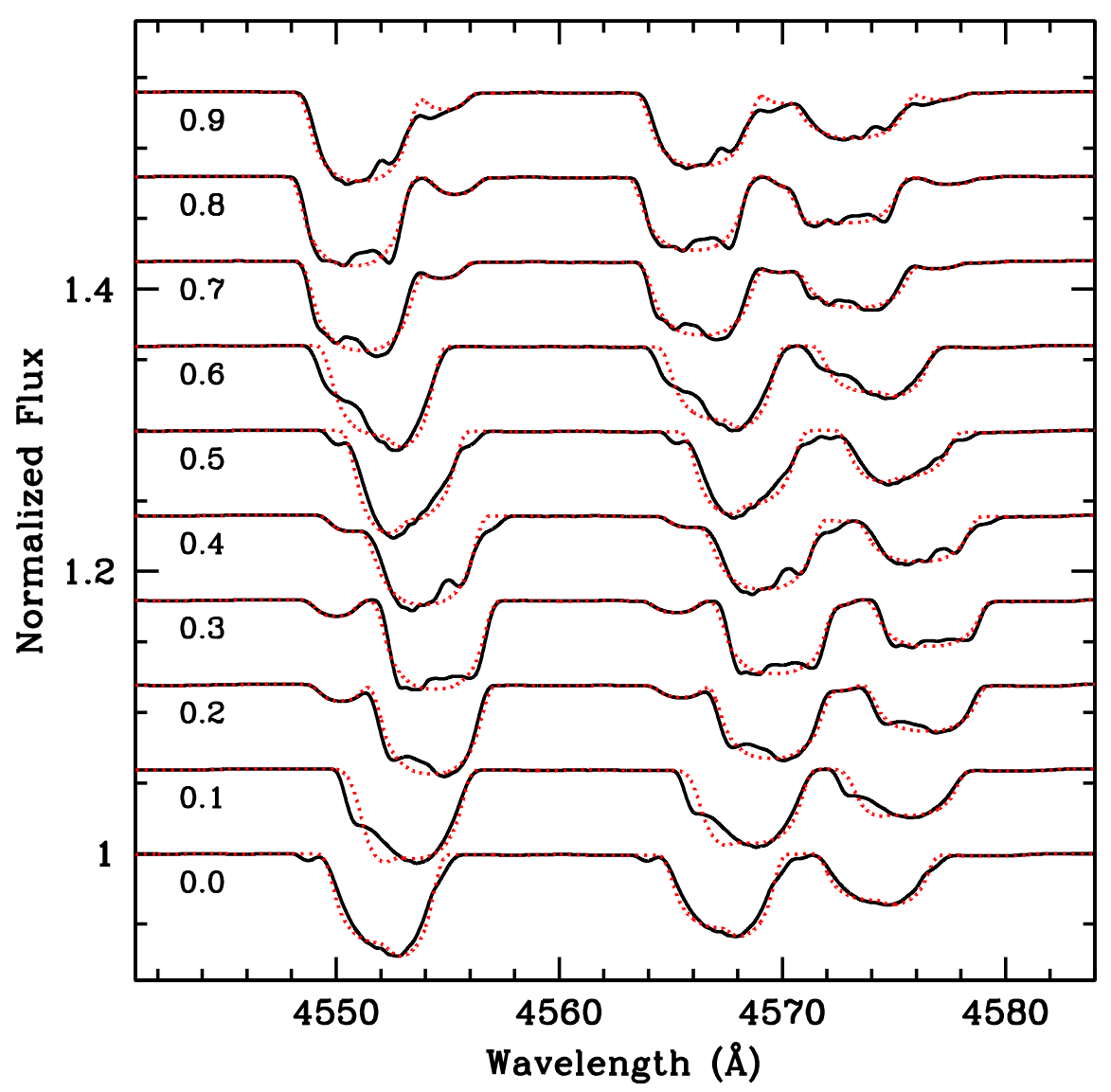

Fig. 2. The synthetic primary + secondary combined spectra of the Si III triplet are stacked in order of increasing orbital phase ( $\phi=0$ corresponds to periastron). The perturbed spectra are displayed with the dark tracing and the unperturbed spectra with dots. The "bumps" in the primary star's perturbed profiles are evident as is the difficulty they introduce in properly locating the contribution of the secondary except at $\phi \sim 0.3 \pm 0.05$ and $0.8 \pm 0.05$ when the contribution from the secondary is clearly resolved.

CoMBiSpeC. Fig. 2 shows the Si III triplet line profiles at 10 orbital phases in the perturbed and the unperturbed spectra ${ }^{1}$. The strong phase-dependent variations in the perturbed profiles due to tidal interaction are clearly seen and are similar to those present in the observational data. The same behaviour is present in numerous other photospheric

\footnotetext{
${ }^{1}$ Perturbed and unperturbed spectra stand respectively for models with and without tidal interaction.
} 
absorptions without the need of any assumption regarding non-radial pulsations.

\section{Conclusion}

CoMBiSpeC is a first step in the spectral modelling of massive binaries. This model includes various effects like reflection, radiation pressure, gravity darkening, limb-darkening that allow us to compute physical properties $\left(T_{\text {eff }}, \log g\right)$ at the stellar surface and, from there the spectra of binary systems. We have presented here two effects that are now explained in a rather simple way: the temperature distribution effect and the S-S effect. If we combine CoMBiSpeC and the TIDES code, we are able to compute the spectra of eccentric binaries. This 2-in-1 model has lead us to provide a new explanation to the line profile variation of Spica that come only from tidal response of the surface of the primary star. Several improvements could also be done: extension to non-solar abundances, different turbulent velocity, lines affected by stellar winds, "cross-talk" (i.e. heat transfert) between the surface elements, new treatment of the reflection effect, extension to low mass star,... However, despite these limitations and assumptions, our results are encouraging, rendering CoMBiSpeC a interesting tool for the analysis of massive binaries.

I would like to acknowledge Gregor Rauw for a careful reading of this paper and helpful comments. I also wish to express my gratitude to Gloria Koenigsberger for guidance in the use and implementation of the TIDES code. Finally, I acknowledge support through the XMM/INTEGRAL PRODEX contract (Belspo), from the Fonds de Recherche Scientifique (FRS/FNRS).

\section{References}

Claret, A., \& Bloemen, S. 2011, A\&A, 529, 75

Drechsel, H., Haas, S., Lorenz, R., \& Gayler, S. 1995, A\&A, 294, 723

Harrington, D.M., Koenigsberger, G., Moreno, E., \& Kuhn, J. 2009, ApJ, 704, 813

Howarth, I. D. 1997, Observatory, 117, 335

Lanz, T., \& Hubeny, I. 2003, ApJS, 146, 417

Lanz, T., \& Hubeny, I. 2007, ApJS, 169, 83

Linder, N., Rauw, G., Sana, H., De Becker, M., \& Gosset, E. 2007, A\&A, 474, 193

Moreno, E. \& Koenigsberger, G. 1999, Rev. Mex. Astron. Astrofis., 35, 157

Moreno, E., Koenigsberger, G. , \& Toledano, O. 2005, A\&A, 437, 641

Moreno, E., Koenigsberger, G., \& Harrington, D. M. 2011, A\&A, 528, 48

Palate, M., \& Rauw, G. 2012, A\&A, 537, 119

Palate, M., Rauw, G., Koenigsberger, G., \& Moreno E. 2012, A\&A, 552, 39

Shobbrook, R.R., Herbison-Evans, D., Johnson, I.D. \& Lomb, N.R. 1969, MNRAS, 145, 131

Shobbrook, R.R., Lomb, N.R., \& Herbison-Evans, D. 1972, MNRAS, 156, 165

Palate, M., Koenigsberger, G., Rauw, G., Harrington, D., \& Moreno, E. 2013, A\&A, 556, 49

Phillips, S.N., \& Podsiadlowski, Ph. 2002, MNRAS, 337, 431

von Zeipel, H. 1924, MNRAS, 84, 665

Wilson, R.E. 1990, ApJ, 356, 613 